Provided for non-commercial research and education use. Not for reproduction, distribution or commercial use.

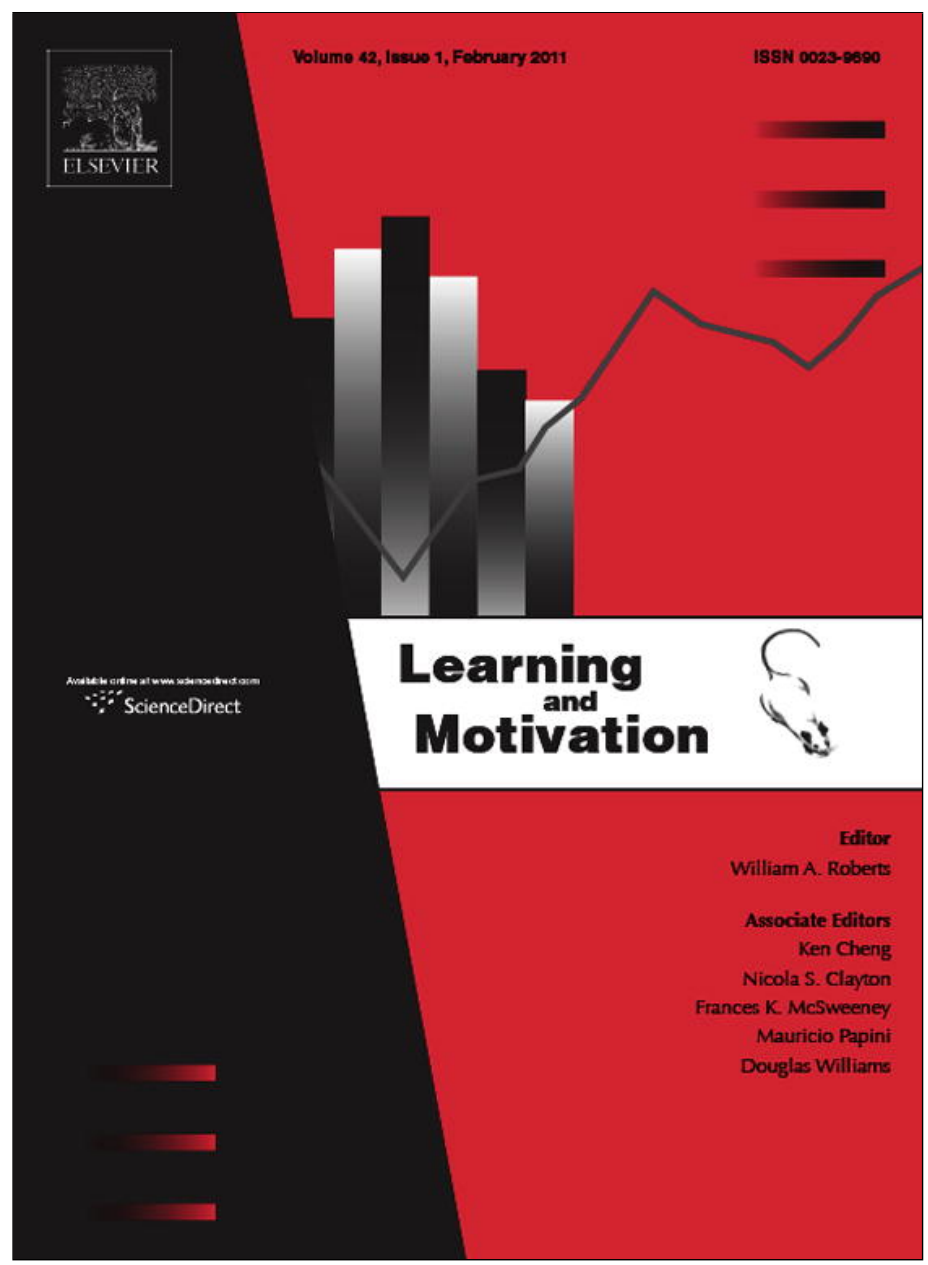

This article appeared in a journal published by Elsevier. The attached copy is furnished to the author for internal non-commercial research and education use, including for instruction at the authors institution and sharing with colleagues.

Other uses, including reproduction and distribution, or selling or licensing copies, or posting to personal, institutional or third party websites are prohibited.

In most cases authors are permitted to post their version of the article (e.g. in Word or Tex form) to their personal website or institutional repository. Authors requiring further information regarding Elsevier's archiving and manuscript policies are encouraged to visit:

http://www.elsevier.com/copyright 


\title{
Psychological distance to reward: Effects of S+ duration and the delay reduction it signals
}

\author{
Jérôme Alessandri ${ }^{\mathrm{a}, *}$, Stephanie Stolarz-Fantino ${ }^{\mathrm{b}}$, Edmund Fantino $^{\mathrm{b}}$ \\ a University of Lille 3, France \\ b University of California, San Diego, United States
}

\section{A R T I C L E I N F O}

\section{Article history:}

Received 9 November 2009

Received in revised form 24 June 2010

Accepted 24 June 2010

Available online 3 August 2010

\section{Keywords:}

Preference

Choice

Segmentation

Psychological distance

Delay-reduction theory

Time schedules

Concurrent-chains

Humans

\begin{abstract}
A B S T R A C T
A concurrent-chains procedure was used to examine choice between segmented (twocomponent chained schedules) and unsegmented schedules (simple schedules) in terminal links with equal inter-reinforcement intervals. Previous studies using this kind of experimental procedure showed preference for unsegmented schedules for both pigeons and humans. In this study, two changes in the experimental procedure were made relative to the usual experimental procedure reported in the literature. The first was that in the segmented schedule the second terminal-link stimulus appeared close to reinforcement presentation and the second was that the change in stimulus was brief, allowing the first stimulus to reappear and to be contiguous with reinforcement. With these changes, preference occurred for the segmented schedule. This result is consistent with principles of conditioned reinforcement, including delay-reduction theory.
\end{abstract}

(C) 2010 Elsevier Inc. All rights reserved.

A number of studies with pigeons and humans have shown that in a concurrent-chains schedule, breaking up a simple schedule of reinforcement into more than one component or segment in the terminal link of the chain - each component correlated with a discriminative stimulus - results in less preference for this segmented schedule than for a simple unsegmented schedule with the same overall duration (in pigeons, Duncan \& Fantino, 1972; Leung \& Winton, 1986, 1988; and in humans, Leung, 1989, 1993). A preference for the unsegmented schedule was observed in all these studies whatever the kind of schedule used [e.g., segmented fixed-interval (FI) FI schedules in Duncan and Fantino (1972), segmented variable-interval (VI) VI schedules in Leung (1993), segmented FI fixed-time (FT) schedules in Leung and Winton (1986), and segmented FT FT schedules in Leung (1989)]. Furthermore, when human participants were asked to estimate the length of the segmented and the unsegmented schedules, they overestimated the duration of the segmented alternative (Leung, 1989).

These results from choice procedures are consistent with the literature on extended chain and tandem schedules (e.g., see Fantino, 2008), including Gollub's (1958) landmark study in which pigeons' responding came to a halt in extended chain schedules. The stimuli associated with the early links of the chain were not conditioned reinforcers and did not maintain responding. However, subsequent research showed that when the sequence of stimuli in the chain was alternated, rather than fixed (e.g., instead of a red, blue, and yellow stimuli fixed sequence, a sequence could be blue, red, yellow, or yellow, red, blue), responding in the first component of a three-component FI 1.5 min schedule was well maintained (Kelleher \& Fry, 1962), presumably since all three stimuli were frequently correlated with reinforcement.

\footnotetext{
* Corresponding author at: Department of Psychology, University of Lille Nord de France, rue du Barreau - BP 60149, 59653 Villeneuve d'Ascq Cedex, France.

E-mail address: jerome.alessandri@univ-lille3.fr (J. Alessandri).
} 
Whereas all of the studies cited above support the notion that unsegmented schedules should be preferred to their segmented counterparts, there is one feature of these comparisons that might lead to the opposite prediction. Namely, appearance of the final stimulus in the segmented schedule is generally correlated with more reduction in time to reward ("delay reduction", Fantino, 1969) than the other stimuli in the situation and should serve as a conditioned reinforcer (or "S+"). In the prior comparisons of segmented and unsegmented outcomes, entry into the segmented outcome was always associated with a stimulus never temporally contiguous with reinforcement (an "S-delta"). Instead, if both outcomes were comparable in that the stimulus that occurred at the onset of the terminal link was contiguous in time with reinforcement for both unsegmented and segmented outcomes, then the greater conditioned reinforcement associated with the segmented outcome might result in preference for that outcome. Thus, in the present experiments, conditions were included in which the stimulus presented at the onset of the segmented outcome was also presented before reinforcement. Specifically, during the segmented outcome a stimulus change occurred at some point that, depending on the condition, was correlated with different degrees of delay reduction. Given a constant overall duration of the segmented outcome, the shorter the interval between the onset of the brief stimulus and reinforcement, the greater the delay-reduction correlated with that stimulus. After termination of this stimulus, the stimulus that had introduced the segmented outcome was reinstated and remained present until reinforcement presentation.

Our first hypothesis is that, when the stimulus occurring at the onset of both segmented and unsegmented outcomes is the same as that occurring just before reinforcement, then preference for the unsegmented outcome, consistently reported in prior studies, would no longer be evident since in this case both stimuli should be conditioned reinforcers. Our second hypothesis is that the delay-reduction correlated with the brief stimulus occurring in the segmented outcome should actually result in preference for that alternative as it should serve as an additional source of conditioned reinforcement. Moreover, the greater the delay-reduction, the greater the preference should be. Our third hypothesis is that we should replicate prior findings of preference for unsegmented outcomes when the comparison is between an unsegmented outcome and a segmented schedule in which the stimulus occurring at the onset of the segmented outcome is different from the stimulus preceding reinforcement. Note that there are two sources of conditioned reinforcement in these procedures. One involves stimuli at the initiation of the terminal link that are also associated with reinforcement (and which represent delay reduction with respect to the preceding initial links). The second is the additional delay-reduction correlated with the onset of the brief stimulus during the terminal link. As we shall see, both contribute to preference.

\section{Experiment 1}

In this experiment preference for a variety of segmented schedules (described below) was compared with preference for unsegmented schedules of the same total length.

\section{Method}

\section{Participants}

The participants were 120 undergraduate students (about 70\% women and $30 \%$ men) at the University of Lille III who were all volunteers. No course credit or money was given for participation.

\section{Apparatus}

The study was conducted in a small room. Each participant was seated on a chair at a table on which there was a monitor connected to a computer. All participants were trained and tested with a program created with Labview 8.0 (National Instruments Corporation, Austin, TX).

\section{Procedure}

The procedure involved presentation of a concurrent-chains schedule with two alternatives. Each session consisted of 8 presentations (except for the 50\% chain-like 14 trials condition, see below) of a block consisting of two forced trials (i.e., one for each alternative) and one test trial. On the forced trials, only one initial-link stimulus was presented at a time; this stimulus, a white circle, was presented either at the left or right side of the monitor screen. One mouse click to the presented circle made it disappear (i.e., fixed-ratio 1 or FR1) and initiated the terminal-link stimulus: a change in the color of the circle (i.e., green or blue, see the different conditions for a detailed description). In all conditions conducted in this experiment, the schedule of reinforcement was segmented FR1 FT 30 s (i.e., reinforcement delivered after 30 s independent of any response). For the unsegmented alternative, there was no change in the color of the circle during the terminal link until reinforcement delivery. For the segmented alternative there were changes in the color of the circle as shown on the left side of Fig. 1. For both alternatives, the reinforcer was a $1 \mathrm{~s}$ presentation of a preferred picture chosen previously by the participant from among 15 available pictures of popular paintings and landscapes. The order of forced trial type (i.e., segmented or unsegmented alternative) was randomized within participants, and the side at which segmented and unsegmented alternatives appeared was counterbalanced across participants. On choice trials, a concurrent-chains schedule 


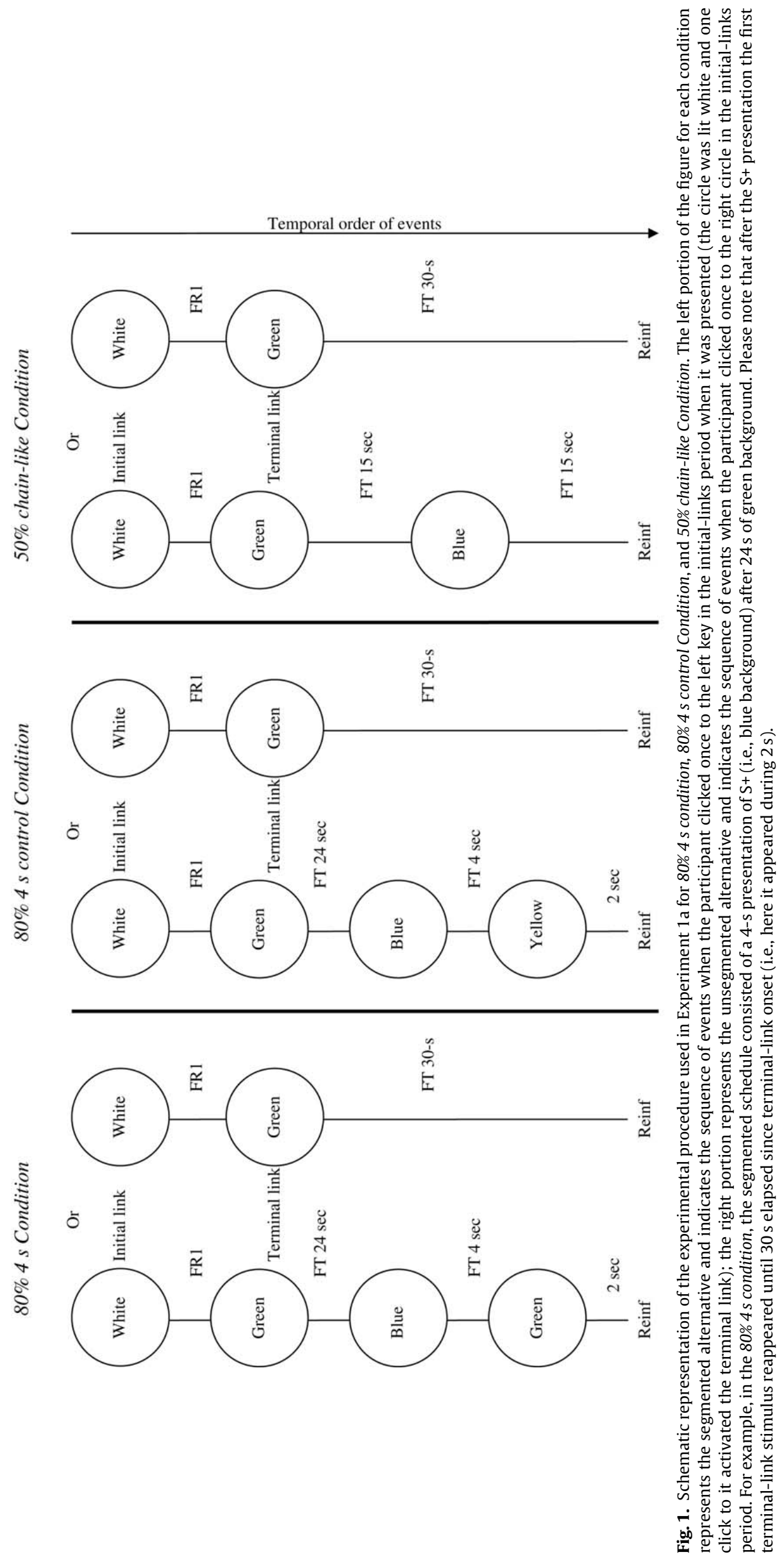


was in effect. Initially the participant had to click on a circle presented at the middle of the monitor screen. This click was followed by the simultaneous presentation of the two corresponding circles presented previously on forced trials. One click to either the right or the left circle made both circles disappear and initiated the terminal link of the alternative chosen. At the start of each session participants were given the following instructions (in French) presented on the screen before the forced trials:

"You will see the picture you have selected presented on the monitor screen. To begin each presentation of the picture you have to click on one circle that will be presented at different locations on the screen. You will have to wait a few seconds before picture presentation. During this time please remain focused to the screen because you could miss a picture. Please do not count the time during the waiting period."

And before the first choice trial the following instructions were given:

"The task is the same as previously except that after having clicked on the circle presented at the middle of screen two circles will appear and you have to choose the one you prefer by clicking on it to see the picture. The choice you will make will have no effect on the following trial type, nor on the remaining number of trials that were programmed in advance. There are no good and bad responses. And please choose as quickly and spontaneously as you can. If not your choice response won't be recorded."

Participants were also instructed orally to pay attention to differential outcomes following each choice. Also participants were told "to remain focused on the circle because events that will appear on the different sides will be different". The experiment was completed in one session of about $25 \mathrm{~min}$. The percentage of choices for the segmented alternative over the last 4 test trials was calculated and analyzed. Each student participated in only one of the following conditions:

50\% chain-like condition: The initial-links stimuli were presented at the right and at left of the monitor screen. During the terminal link, on the unsegmented side there was no S+ presentation or any further change in the circle's color, which remained blue or green (counterbalanced across participants) during the entire terminal link; on the segmented (chain-like) side, there was a further change in color (i.e., S+ presentation) after $15 \mathrm{~s}$ that remained for another $15 \mathrm{~s}$ until reinforcement. The sequence of stimuli for the segmented schedule could be either blue-green or green-blue, counterbalanced across participants.

50\% chain-like 14 trials condition: This condition was similar to the 50\% chain-like condition except that 14 trials were carried out in order to assess whether the results would be the same for 14 trials as with 8 trials, the number of trials conducted in all other conditions.

$50 \% 4 \mathrm{~s}$ condition: This condition was similar to the $50 \%$ chain-like condition except that the change in the color of the circle remained for $4 \mathrm{~s}$, and the first terminal link stimulus was reinstated during $2 \mathrm{~s}$ until reinforcement (resulting in either a green-blue-green sequence or a blue-green-blue sequence).

$80 \%$ chain-like condition: This condition was similar to the $50 \%$ chain-like condition except that for the segmented alternative the change in color did not occur until after $80 \%$ of the terminal link had transpired $(24 \mathrm{~s}$, compared to $15 \mathrm{~s}$ in the $50 \%$ chain-like conditions).

$80 \% 4$ s condition: This condition was similar to the $50 \%$ chain-like condition except that, for the segmented alternative, the change in color of the circle occurred after $24 \mathrm{~s}$, remained for $4 \mathrm{~s}$, and the first terminal link stimulus was reinstated during $2 \mathrm{~s}$ until reinforcement (resulting in either a green-blue-green sequence or a blue-green-blue sequence).

$80 \% 4$ s control condition: This condition was similar to the $80 \% 4 \mathrm{~s}$ condition except that for the segmented alternative the first terminal link stimulus was not reinstated after the second terminal link stimulus; instead, another stimulus (change to yellow) was presented during the final $2 \mathrm{~s}$ until reinforcement.

Twenty naïve participants were recruited for each condition.

\section{Results and discussion}

The results for individual participants in each condition described above, shown as the percentage of choices for the segmented alternative, appear in Table 1 . Several statistical analyses were conducted; in all cases, alpha was set at .05. First, a two-way ANOVA was conducted in order to test the effect of two factors: reinstatement (which compared the $80 \% 4 \mathrm{~s}$ and the $50 \% 4 \mathrm{~s}$ with the $80 \%$ chain-like and $50 \%$ chain-like schedules); and delay reduction (which compared the $80 \%$ chain-like and $80 \% 4 \mathrm{~s}$ with the $50 \%$ chain-like and the $50 \% 4 \mathrm{~s}$ schedules), as well as their interaction. The Reinstatement factor tested the hypothesis that - when the stimulus occurring at the onset of both segmented and unsegmented outcomes is the same as that occurring just before reinforcement - preference for the segmented outcome will increase. The delay-reduction factor tested the second hypothesis - that the greater the delay-reduction correlated with the brief stimulus, the greater the preference for that alternative. There were significant main effects of Reinstatement, $F(1,76)=4.28$, and of delay reduction, $F(1,76)=4.28$, and no significant reinstatement $\times$ delay-reduction interaction, $F(1,76)=1.07$. Thus, the results support our first and second hypotheses. Next, we compared preference scores in the $80 \% 4 \mathrm{~s}$ condition and in the $80 \% 4 \mathrm{scontrol}$ condition, in which a novel stimulus was presented during the last $4 \mathrm{~s}$; this analysis revealed a significantly greater preference for the segmented alternative in the $80 \% 4 s$ condition, $t(38)=2.02$, giving further support to the importance of reinstatement of the original stimulus. 
Table 1

Individual participants' percentages of choice for the segmented alternative over the last 4 trials in each condition of Experiment 1a. The groups' mean choice percentages and the standard error of the mean for each condition are also shown.

\begin{tabular}{|c|c|c|c|c|c|c|}
\hline \multirow[t]{2}{*}{ Participant number } & \multicolumn{6}{|c|}{ Condition } \\
\hline & $80 \% 4 \mathrm{~s}$ & $80 \% 4$ s control & $80 \%$ chain-like & $50 \%$ chain-like & $50 \% 4 \mathrm{~s}$ & $\begin{array}{l}50 \% \text { chain-like } 14 \\
\text { trials } 14 \text { trials }\end{array}$ \\
\hline 1 & 75 & 50 & 50 & 25 & 50 & 50 \\
\hline 2 & 50 & 0 & 50 & 0 & 0 & 25 \\
\hline 3 & 75 & 50 & 100 & 75 & 100 & 0 \\
\hline 4 & 100 & 25 & 75 & 0 & 75 & 50 \\
\hline 5 & 50 & 0 & 0 & 50 & 75 & 0 \\
\hline 6 & 25 & 100 & 50 & 25 & 50 & 100 \\
\hline 7 & 25 & 75 & 75 & 75 & 50 & 50 \\
\hline 8 & 75 & 50 & 100 & 25 & 75 & 75 \\
\hline 9 & 75 & 75 & 25 & 50 & 100 & 0 \\
\hline 10 & 75 & 50 & 50 & 25 & 25 & 25 \\
\hline 11 & 75 & 25 & 75 & 75 & 25 & 0 \\
\hline 12 & 100 & 100 & 50 & 50 & 100 & 100 \\
\hline 13 & 50 & 50 & 50 & 25 & 25 & 100 \\
\hline 14 & 25 & 50 & 75 & 75 & 50 & 0 \\
\hline 15 & 100 & 50 & 100 & 50 & 100 & 0 \\
\hline 16 & 75 & 0 & 75 & 25 & 75 & 0 \\
\hline 17 & 75 & 50 & 75 & 50 & 75 & 0 \\
\hline 18 & 100 & 50 & 25 & 50 & 50 & 75 \\
\hline 19 & 25 & 50 & 50 & 75 & 25 & 50 \\
\hline 20 & 75 & 75 & 50 & 0 & 75 & 100 \\
\hline Mean & 66.25 & 48.75 & 60 & 41.25 & 60 & 40 \\
\hline SEM & 5.81 & 6.41 & 5.85 & 5.81 & 6.64 & 8.96 \\
\hline
\end{tabular}

The results do not support our third hypothesis - that we should replicate preference for unsegmented outcomes found in prior research (e.g., Leung, 1989, 1993). Specifically, when comparing an unsegmented outcome with a segmented outcome in which the stimulus occurring at the onset of the segmented outcome is different from the stimulus preceding reinforcement, there should be preference for the unsegmented outcome (although preference was in the correct direction - 59\% for the unsegmented outcome in the 50\% chain-like condition). Finally, no difference was observed in preference scores between the 50\% chain-like condition (mean preference 41.25 for the segmented alternative) and the 50\% chain-like 14 trials condition (mean preference 40.0), showing that there was no particular benefit to conducting a greater number of trials in order to determine preference.

\section{Experiment 2}

In this study, participants were exposed to a direct comparison between two segmented schedules - 80\% $4 \mathrm{~s}$ segmented and $50 \% 4 \mathrm{~s}$ segmented - differing in the degree of delay reduction that was associated with the appearance of the change in color that occurred during the terminal link. The color change in the $80 \%$ segmented schedule was associated with a greater reduction in time to reinforcement than that occurring in the 50\% segmented schedule. Thus, according to delay-reduction theory, there should be preference for the $80 \%$ segmented schedule.

\section{Method}

Participants

This condition was tested with 16 naïve participants.

\section{Procedure}

The experimental procedure used here was similar to that of Experiment 1 except for the terminal-links events; now, both alternatives were segmented, one at $50 \%$ and the other at $80 \%$ of the terminal-link duration. The terminal-link duration was the same for both conditions. For the $50 \%$ segmented alternative the change in stimulus occurred after $15 \mathrm{~s}$ and for the $80 \%$ segmented alternative the change in stimulus occurred after $24 \mathrm{~s}$. The S+ duration was $4 \mathrm{~s}$ for both alternatives. Again, the first terminal link stimulus was reinstated following the $S+$ presentation and lasted until 30 s had elapsed since the entry into the terminal link. 
Table 2

Individual participants' percentages of choice for the alternative segmented at $80 \%$ (compared to an alternative segmented at $50 \%$ ) on the last 4 trials in Experiment 2. The group's mean choice percentage and the standard error are also shown. An asterisk shows a significant effect of preference for the $80 \%$ alternative.

\begin{tabular}{rc}
\hline Participant number & Choice percentage \\
\hline 1 & 25 \\
2 & 75 \\
3 & 75 \\
4 & 50 \\
5 & 100 \\
6 & 50 \\
7 & 50 \\
8 & 0 \\
9 & 100 \\
10 & 100 \\
11 & 75 \\
12 & 50 \\
13 & 75 \\
14 & 50 \\
15 & 75 \\
16 & 100 \\
Mean & $65.62^{*}$ \\
SEM & 7.17 \\
\hline
\end{tabular}

\section{Results and discussion}

Table 2 presents results from Experiment 2. An analysis of the last four trials showed a significant preference for the $80 \%$ segmented schedule over the $50 \%$ segmented schedule, $t(15)=2.18$, supporting the role of delay reduction. Of the 11 participants who showed a preference, 9 preferred the $80 \%$ segmented schedule.

\section{General discussion}

The present results show that under certain conditions some humans preferred a segmented schedule to an unsegmented schedule. In particular, two variables appeared to play an important role in affecting the relative value of the segmented schedule. The first variable was the interval between the second stimulus onset (or offset) and reinforcement onset in the segmented alternative or the degree of delay-reduction correlated with the onset of the brief stimulus. When the stimulus was correlated with an $80 \%$ reduction in time to reinforcement, preference for the segmented outcome was significant. When the stimulus was correlated with only a $50 \%$ reduction in time to reinforcement, however, a significant preference was not obtained (see Table 1). In addition, when the $50 \%$ and $80 \%$ conditions were directly compared, preference was obtained for the $80 \%$ delay-reduction outcome (Table 2 ). This finding is consistent with delay-reduction theory (Fantino, 1969).

The second influential variable was whether or not the stimulus that occurred at the outset of the outcome phase was reinstated after the brief presentation of the $\mathrm{S}+$. That is, significant preference for the segmented schedule occurred only when the first stimulus of the outcome phase was also correlated with reinforcement (i.e., $80 \% 4 \mathrm{~s}$ condition); when the first stimulus was not reinstated ( $80 \% 4 \mathrm{~s}$ control condition) no preference occurred.

We should address the relation of the present results to the extensive literature on choice for unsegmented schedules over segmented ones, reviewed in the Introduction. The preference for segmented schedules found under certain temporal conditions in the present series of experiments may be attributed to the fact that the same stimulus present at the onset of the segmented outcome was also contiguous with reinforcement. The stimulus present at the onset of the segmented outcome in the prior research was always far removed, temporally, from reinforcement and might be considered an S-Delta (e.g., Duncan \& Fantino, 1972). The discrepancy between the present results and the prior research comes from the lack of preference for the unsegmented outcome in the 50\% chain-like conditions. But looking carefully at the prior results, especially in humans published by Leung $(1989,1993)$, we see that the preference for the unsegmented schedule was relatively small but with less inter-subject variability (i.e., around a mean 55-60\%). A review of data from Table 1 shows that in both conditions that replicated Leung's design (i.e., 50\% chain-like and 50\% chain-like 14 trials), the mean choice proportion favored the unsegmented outcome (i.e., about 60\%) by the same degree as in Leung's studies. However, in these cases there is more variability between participants. Specifically, some participants preferred the segmented outcome ( $25 \%$ of the participants) but no one did in Leung's studies. In any event, preference for the segmented outcome was clearly eliminated.

A different picture emerges from the $80 \%$ chain-like results. Here there is actually a preference in the direction of the segmented outcome (60\%), though here again there was high variability between participants. We conjecture that the $80 \%$ delay-reduction correlated with the brief stimulus inserted into the segmented outcome adds enough conditioned reinforcement value to overcome the advantage of the unsegmented outcome. 
We have interpreted lack of preference for the unsegmented outcome in terms of the advantage conferred by the fact that the first stimulus is contiguous with reinforcement delivery, in both outcomes, a feature not present in prior studies comparing segmented and unsegmented outcomes. However, in order to explain preference for the segmented outcome, we need an additional account, also in terms of conditioned reinforcement. The brief stimulus ( $\mathrm{S}+$ ) may confer added value to the segmented outcome by virtue of its temporal (and/or signalling) relation to the presentation of reinforcement. This explanation is consistent with one given by Schaal and Branch (1990) in their study of "delay signals". According to this account, the S+ signals a temporal relation to reinforcer presentation that is absent in the corresponding unsegmented outcome. Three sets of studies are consistent with this account. First, we have the present results in which segmented outcomes are favored and in which how much they are favored depends on the degree to which the S+ is correlated with a reduction in time to reinforcement. Second, we have the results of Schaal and Branch (1990). They investigated rates of responding in VI 60-s schedules of 27-s delayed reinforcement. They assessed the role of a delay signal that varied between $0.5 \mathrm{~s}$ and $27 \mathrm{~s}$ (the entire delay interval). They found that the longer the delay signal $(\mathrm{S}+)$ the higher the response rates generated on the VI 60-s schedule. When the delay signal was cotemporaneous with the entire delay ( $27 \mathrm{~s}$ ), of course, the schedule was unsegmented and the high response rates are in keeping with the traditional preference for unsegmented over segmented outcomes. In terms of the present results, the comparability lies in the fact that the longer the S+ the closer was its offset to primary reinforcement, as in the $80 \%$ versus $50 \%$ delay-reduction comparisons of the present experiments (see in contrast the rate-decreasing effect when the delay was not signaled, Sizemore \& Lattal, 1977, 1978). The third sets of experiments that are consistent with this account in terms of the conditioned-reinforcing value of the S+ have been reviewed recently by Fantino and Romanowich (2007) and Fantino (2008). When putative conditioned reinforcers are superimposed on the outcome schedules of concurrent-chains schedules but these superimposed stimuli are not correlated with a reduction in time to reinforcement, they do not enhance preference (e.g., Schuster, 1969; Squires, 1972). Only stimuli correlated with a reduction in time to primary reinforcement (or, in Mazur's terms, an increase in value; Mazur, 2001) are bona fide conditioned reinforcers and only those stimuli enhance preference.

In summary, the present studies show a preference for a segmented schedule over an unsegmented one. The experiments have identified two necessary though not sufficient conditions to obtain such a preference: the first is that in the segmented alternative, the $\mathrm{S}+$ onset (and especially offset) needs to be relatively close to reinforcement onset, and the second is that the first terminal-link stimulus should reappear and be contiguous with reinforcement. We have suggested that the preference for segmented schedules in these conditions is due to the role played by the conditioned reinforcer $(\mathrm{S}+)$ added to the segmented alternative.

\section{References}

Duncan, B., \& Fantino, E. (1972). The psychological distance to reward. Journal of the Experimental Analysis of Behavior, $18,23-34$.

Fantino, E. (1969). Choice and rate of reinforcement. Journal of the Experimental Analysis of Behavior, 12, 723-730.

Fantino, E. (2008). Choice, conditioned reinforcement, and the Prius effect. The Behavior Analyst, 31, 95-111.

Fantino, E., \& Romanowich, P. (2007). The effect of conditioned reinforcement rate on choice: A review. Journal of the Experimental Analysis of Behavior, 87, 409-421.

Gollub, L. R. (1958). The chaining of fixed-interval schedules. Unpublished doctoral dissertation. Harvard University.

Kelleher, R. T., \& Fry, W. T. (1962). Stimulus functions in chained fixed-interval schedules. Journal of the Experimental Analysis of Behavior, 5, $167-173$.

Leung, J. P., \& Winton, A. S. W. (1986). Preference for less segmented schedules fixed-time components in concurrent-chain schedules of reinforcement. Journal of the Experimental Analysis of Behavior, 46, 175-183.

Leung, J. P., \& Winton, A. S. W. (1988). Preference for simple interval schedules of reinforcement in concurrent chains: Effect of segmentation ratio. Journal of the Experimental Analysis of Behavior, 49, 9-20.

Leung, J. P. (1989). Psychological distance to reward: A human replication. Journal of the Experimental Analysis of Behavior, 51, $343-352$.

Leung, J. P. (1993). Psychological distance to reward: Segmentation of aperiodic schedules of reinforcement. Journal of the Experimental Analysis of Behavior, $59,401-410$

Mazur, J. E. (2001). Hyperbolic value addition and general models of animal choice. Psychological Review, 108, 96-112.

Schaal, D. W., \& Branch, M. N. (1990). Responding of pigeons under variable-interval schedules of signaled-delayed reinforcement: Effects of delay-signal duration. Journal of the Experimental Analysis of Behavior, 53, 103-121.

Schuster, R. H. (1969). The strength of conditioned reinforcers as a function of frequency and probability of reinforcement. In D. P. Hendry (Ed.), Conditioned reinforcement (pp. 192-234). Homewood, IL: Dorsey Press.

Sizemore, O. J., \& Lattal, K. A. (1977). Dependency, temporal contiguity, and response-independent reinforcement. Journal of the Experimental Analysis of Behavior, 27, 119-125.

Sizemore, O. J., \& Lattal, K. A. (1978). Unsignalled delay of reinforcement in variable-interval schedules. Journal of the Experimental Analysis of Behavior, 30, $169-175$.

Squires, N. (1972). Preference for conjoint schedules of primary reinforcement and brief-stimulus presentation. Unpublished doctoral dissertation. San Diego: University of California. 